\title{
Carbon nanotubes in drug delivery: just a carrier?
}

\section{Marta Roldo}

School of Pharmacy and Biomedical Science, University of Portsmouth, St Michael's Building, White Swan Road, Portsmouth PO1 2DT. Tel: 00442392843586; Fax: 00442392843565; e-mail: marta.roldo@ port.ac.uk

Author photographs: suitable high-resolution head shot for inclusion.

Key words: Carbon nanotubes; drug delivery; drug targeting.

Iijima's 1991 discovery brought to the attention of the research community a new allotrope of carbon: carbon nanotubes (CNTs). Since then CNTs have been extensively studied and have demonstrated to be of great potential in various industrial and biomedical fields of application [1].

CNTs can be described as rolled-up sheets of graphene forming single or multi walled seamless cylinders; their diameters range from few to hundreds of nanometres, respectively. They generally present a length/diameter ratio higher than $10^{6}$ and have exceptional electrical, thermal, mechanical and optical properties [2,3]. Notwithstanding their great potential, the observed toxicity of these novel materials caused major concerns. CNTs are highly hydrophobic in nature and tend to form bundles in aqueous environments, leading to unstable dispersions. Bundles are phagocyted and cause cellular necrosis and oxidative stress damage. Furthermore, their characteristic shape, similar to asbestos fibres, is responsible for pulmonary toxicity that results in granulomas and fibrosis [4]. However, great advances have been achieved in the last decades in improving the water compatibility and reducing the toxicity of CNTs via both covalent and noncovalent modification [5]. The strategies developed have allowed researchers to investigate numerous possible biomedical applications, including their use as drug carriers for anticancer drugs, gene therapy and as scaffold components in tissue engineering [4].

\section{Carbon nanotubes as drug carriers}

The properties that make CNTs ideal drug carriers are multiple. CNTs have a high specific surface area per unit weight, offering enhanced loading capacity compared to conventional nanomaterials of spherical shape [6]. They have been used for the delivery of 
several anticancer drugs, with doxorubicin (DOX) being the most extensively studied. The possibility of forming $\pi-\pi$ interactions between DOX and the surface of CNTs makes loading simple and efficient; furthermore, the high surface area offered by CNTs allows for a high degree of loading that cannot be achieved by other carrier systems. Using a non-covalent loading mechanism means that loading is not limited to type and number of functional groups present on the drug molecule and the carrier; moreover it allows for the loading of other lipophilic aromatic compounds (e.g. daunorubicin, camptothecin) [7]. Furthermore, $\mathrm{pH}$ dependent loading and release can be achieved so that the system can provide site specific release of the drug, thus increasing efficacy and reducing toxicity [8,9]. Studies on targeted drug delivery have now been conducted for over a century, since Ehrlich developed the concept of the "Magic Bullet" in 1906 [10]. Many strategies have been employed from active targeting, by conjugation of monoclonal antibodies or smaller moieties recognised by a specific receptor (i.e. folate receptor overexpressed by certain types of tumours); to PEGylation and exploitation of the Enhanced Permeability and Retention (EPR) effect. After simple oxidation procedures in the presence of concentrated acids, CNTs provide multiple points amenable to chemical modification allowing for extensive and multiple functionalization [11]. Due to the ease of functionalization, targeting strategies used for polymeric and particulate carriers can been applied also to CNTs. However, a recent study has raised doubts about the possibility of achieving targeted drug delivery to cancer cells by the covalent attachment of folic acid to the surface of CNTs [11]. It is well known that CNTs are able to cross the cell membrane by virtue of their shape [12,13] and this could limit their applications in selective targeting. On the other hand, the literature is rich in examples that testify the contrary; therefore these findings highlight the need for further research to elucidate the role of CNTs in targeted drug delivery. Strategies that employ magnetically driven targeting [14] could offer a good alternative for selective targeting, even though they might result to be more expensive and more difficult to implement in a clinical setting. 
A further advantage in the use of CNTs is afforded by their strong absorption in the near infrared, Raman scattering and photoacoustic properties that can be exploited for imaging purposes providing an ideal tracking system for drug delivery or a diagnostics strategy $[11,15]$. Furthermore, since CNTs can emit heat when irradiated at near infrared wavelengths, their thermal properties have been exploited for causing local hyperthermia in photothermal ablation procedures for cancer therapy [16].

\section{A new application avenue}

Most recently, an increased number of publications regard the potential use of CNTs in vaccine delivery. Initially, interest was sparked by the possibility of loading multiple antigens to the surface of CNTs and by their ability to rapidly internalise into antigen presenting cells [17]. However, more recently, it has become clear that the potential of CNTs is beyond that of an efficient carrier for vaccines. A genome-wide study, carried out comparing CNTs of different diameter and different surface functionalization, has highlighted their potential use as adjuvants and not just carriers for vaccine delivery [18]. This study observed that CNTs are able to boost pathways linked to the early innate response that are normally activated in response to infection, vaccination, transplant rejection and anti-tumour immune response suggesting their potential use in stimulating an effective inflammatory response, pathogen clearance and tumour rejection. CNTs have the potential of exceeding the performance of aluminium hydroxide as a vaccine adjuvant, since the latter is able to enhance the induction of the humoral immune response only, while CNTs have been shown to increase cell-mediated response [18].

\section{A word of caution}

Very elegant, multifunctional, engineered CNTs derivatives and conjugates for drug delivery have been extensively described in literature. Constructs that combine drug loading properties with enhanced biocompatibility, targeting ability and imaging or tracking options have been developed to both exploit the positive and counteract the negative intrinsic properties of CNTs. However, the translational potential of nanomedicine so far has been achieved only by the 
simplest systems. Therefore, a paradigm shift is required; we need to move from sophisticated and elaborated ways of modifying CNTs to more simplified and flexible approaches. For example, favouring non-covalent modification could present an adaptable approach for the functinalisation of different types of CNTs and developing one pot functionalization methods could reduce development time and cost. Furthermore, despite intense research on carbon nanotubes there is still a wealth of unanswered questions about their interaction with the human body, more detailed and systematic studies need to be undertaken in this direction. Clinical applications are still not a reality however strategies to harvest the full potential of carbon nanotubes are continually invented, developed and refined as testified by the wealth of literature regularly published from experts in various biomedical specialities.

\section{References:}

1. Iijima S. Helical microtubules of graphitic carbon. Nature, 354(6348), 56-58 (1991).

2. Gulati N, Gupta H. Two Faces of Carbon Nanotube: Toxicities and Pharmaceutical Applications. Crit. Rev. Ther. Drug Carrier Syst., 29(1), 65-88 (2012).

3. Lu W, Zu M, Byun J-H, Kim B-S, Chou T-W. State of the Art of Carbon Nanotube Fibers: Opportunities and Challenges. Adv. Mater., 24(14), 1805-1833 (2012).

4. Roldo M, Fatouros DG. Biomedical applications of carbon nanotubes. Annual Rep. C (Phys. Chem.), 109, 10 (2013).

5. Fatouros GD, Roldo M, Van der Merwe SM. Stabilisation of carbon nanotube suspensions. In: Carbon Nanotubes: From Bench Chemistry to Promising Biomedical Applications. Pastorin, G (Ed. (Pan Stanford Publishing Pte. Ltd., Singapore, 2011) 122.

6. Zhang W, Zhang Z, Zhang Y. The application of carbon nanotubes in target drug delivery systems for cancer therapies. Nanoscale Res. Lett., 6(1), 1-22 (2011).

7. Liu Z, Fan AC, Rakhra K et al. Supramolecular Stacking of Doxorubicin on Carbon Nanotubes for In Vivo Cancer Therapy. Ang. Chem. Int. Ed., 48(41), 7668-7672 (2009).

8. Chen P, Wang Z, Zong S et al. pH-sensitive nanocarrier based on gold/silver coreshell nanoparticles decorated multi-walled carbon manotubes for tracing drug release in living cells. Biosen. Bioelectron, 75, 446-451 (2016).

9. Zhang X, Meng L, Lu Q, Fei Z, Dyson PJ. Targeted delivery and controlled release of doxorubicin to cancer cells using modified single wall carbon nanotubes. Biomaterials, 30(30), 6041-6047 (2009).

10. Gaynes RP. Germ theory: medical pioneers in infectious diseases (Am Soc Microbiol, 2011).

11. Ménard-Moyon C, Ali-Boucetta H, Fabbro C, Chaloin O, Kostarelos K, Bianco A. Controlled Chemical Derivatisation of Carbon Nanotubes with Imaging, Targeting, and Therapeutic Capabilities. Chem. Eu. J., 21(42), 14886-14892 (2015). 
12. Kraszewski S, Bianco A, Tarek M, Ramseyer C. Insertion of Short AminoFunctionalized Single-Walled Carbon Nanotubes into Phospholipid Bilayer Occurs by Passive Diffusion. PloS one, 7(7), e40703 (2012).

13. Kraszewski S, Picaud F, Elhechmi I, Gharbi T, Ramseyer C. How long a functionalized carbon nanotube can passively penetrate a lipid membrane. Carbon, 50(14), 5301-5308 (2012).

14. Yang F, Jin C, Yang D et al. Magnetic functionalised carbon nanotubes as drug vehicles for cancer lymph node metastasis treatment. Eu. J. Cancer, 47(12), 18731882 (2011).

15. Meng L, Zhang X, Lu Q, Fei Z, Dyson PJ. Single walled carbon nanotubes as drug delivery vehicles: Targeting doxorubicin to tumors. Biomaterials, 33(6), 1689-1698 (2012).

16. Young J, Figueroa E, Drezek R. Tunable Nanostructures as Photothermal Theranostic Agents. Ann. Biomed. Eng., 40(2), 438-459 (2012).

17. Scheinberg DA, McDevitt MR, Dao T, Mulvey JJ, Feinberg E, Alidori S. Carbon nanotubes as vaccine scaffolds. Adv. drug deliv. Rev., 65(15), 10.1016/j.addr.2013.1007.1013 (2013).

18. Pescatori M, Bedognetti D, Venturelli E et al. Functionalized carbon nanotubes as immunomodulator systems. Biomaterials, 34(18), 4395-4403 (2013). 